\title{
5
}

\section{ID-Based Authentication for Mobile Conference Call}

\author{
Shiuh-Jeng Wang, Le-Pond Chin, Jin-Fu Chang \\ Electrical Engineering Department \\ National Taiwan University \\ Taipei, Taiwan 10764 \\ Ywh-Ren Tsai \\ Transmission Technology Laboratory \\ Telecommunication Laboratories \\ Taoyuan, Taiwan 326
}

\begin{abstract}
In this paper a secure conference call setu-up protocol is proposed for a mobile communication system. The proposed scheme uses ID-based concept and enables a mobile unit and a base station to authenticate each other. It has an additional feature of providing mobile subscribers with location privacy. Reauthentication in the course of handoff is also discussed.
\end{abstract}

Keywords

ID-based cryptosystem, privacy, secure protocol, mobile communication

\section{INTRODUCTION}

Wireless has recently become a widely discussed research topic. Many people have already experienced and enjoyed the convenience provided by mobile phone services. With the inclusion of mobile data services in the foreseeable future, we shall march into a a new era of personal multimedia mobile communication services.

Technically speaking, wireless research involves many key issues such as RF technology, propagation, access technique, handoff and channel assignment. Security is also a very important but far less addressed issue in mobile communications.

In wireless communications, signal transmission is done through radio channels and is vulnerable to attacks from intruders. These attacks include avoiding to pay for the use of the system and attempting to gain access to information. These attacks are apparently due to the exposure of the radio link to outsiders. Security has become a great concern of cellular subscribers. To prevent these attacks, tight security measures must be adopted. Authentication is a mechanism designed to expel these illegal attempts. New generation of digital cellular mobile communication such as GSM has made the security improved many orders of magnitude over the analog systems, e.g. Haug (1994).

New generation of wireless system is planned to support a good variety of services such as speech, data, e-mail, fax and etc. According to the technical report, e.g. T1P1.3/93-044R1 (1993), the design objectives of security measures should include: privacy of speech and data, privacy of user location, privacy of call setup information, protection against fraud, protection against disclosure of information, and protection against unauthorized access to subscriber data.

In this paper we concentrate on the construction of an efficient authentication protocol for a mobile communication system. Location privacy is one of our design objectives. Our scheme as a matter of fact provides an additional feature of two-way authentication in the sense that we also allow a subscriber/mobile unit to verify whether a base station is genuine. 
The authentication protocol proposed in this paper is also operable in the course of handoff. What is meant is that during each handoff the base station will be reauthenticated by the original mobile unit, since the base station is changed during handoff in such a way the base station is easily impersonated. In principle, a handoffed mobile needs also to be reauthenticated. In this paper we only propose protocol to reauthenticate a base station. The reauthentication of a mobile station remains to be completed.

In order to meet these requirements, cryptographic techniques should also be carefully chosen. Prior works on security issues in mobile communication systems can be found in Tatebayashi et al. (1990), Hwang (1993), Coomaraswamy and Kumar (1993), Beller et al. (1993), Ashar and Diffie (1994), Lin and Harn (1993) and Molva et al. (1994). Most of these works are done for point-to-point communications. Recently, Hwang and Yang (1995) proposed a public-key-based conference key distribution protocol in which the conference key is shared by all conferees and the authentication is only executed during call set-up.

In this paper, we propose a secure conference call set-up scheme among a group of mobile units via ID-based concept. ID-based cryptoschemes have the convenience of allowing users to communicate secretly using only their identifications. This cryptographic technique has recently received considerable attention from researchers, e.g. Shamir (1985), Okamota and Tanaka (1989) and Tsujii and Itoh (1989). In this paper two authentication protocols are constructed: one for call setup and one for handoff.

In Section II we introduce the principle of ID-based cryptosystem as a preparation. System architecture and protocol are outlined in Section III. Discussions and security analysis are done in Section IV. Conclusions are drawn in Section V.

\section{ID-BASED CRYPTOSYSTEMS}

The well known public key cryptosystem was first proposed by Diffie and Hellman (1976). Public key system allows each user to place his/her encryption key in a public file while keeping the decryption key secret. It has the advantage of requiring no key management. Therefore, the public-key systems are widely used in distributed environment such as a computer network. The ID-based cryptosystem has similar features. It also has an additional feature of not requiring a public file. Any user-associated information such as name, telephone number and address can be used as public key. A smart card ID-based cryptosystem was first proposed by Shamir (1985). we illustrate an ID-based signature scheme as follows. In RSA algorithm, the public keys are $n=p q$ and $e$ while the secret key is $d$, where $p, q$ are two large primes. The encryption key $e$ and decryption key $d$ must satisfy $e d \equiv 1(\bmod (p-1)(q-1))$. Shamir assumes the existence of a key generation center which generates each user $U_{i}$ 's secret key $K_{i}$ by $K_{i}=\left(I D_{i}\right)^{d} \bmod n$, where $I D_{i}$ is $U_{i}$ 's unique identity number, $d$ is a system secret key. In order to sign a message $M$, the smart card computes a pair $(t, s)$ as follows: $t=r^{e} \bmod n$ and $s=K_{i} r^{f(t, M)} \bmod n$, where $r$ is a random number and $f$ is a one-way function. The pair $(t, s)$ is the signature of $M$ which is to be sent to anyone who is the intended recipient of $M$. For legitimate receivers, the signature of $M$ can be easily verified by performing the comparison $s^{e}$ and $I D_{i} t^{f(t, M)}$ in modulo $n$.

In order to realize authentication, the signature scheme is usually used when the communicating parties do not trust each other, e.g. Tanenbaum (1988). In our proposed scheme each mobile unit is equipped with a unique ID. i.e. $I D_{\text {mobile-unit }}$. Each base station is also associated with a unique ID, $I D_{\text {base-station. }}$. Then the authentication is done by validating the 
received signature message.

\section{SYSTEM ARCHITECTURE AND PROTOCOLS}

A cellular mobile communication system consists of three basic parts: subscriber/mobile units (MUs), base stations (BSs) and mobile switching center (MSC). A MU communicates with $\mathrm{BS}$ via the radio link and each $\mathrm{BS}$ is connected to the MSC through the wireline link. To authenticate calls an authentication center (AC) is needed and is assumed to be a part of MSC. The AC of MSC is assumed to have a powerful processor together with a database. Compare to the AC, a BS has rather limited computing power, but it still has a processor to perform high-speed computation. A call setup procedure involves handshakings between a BS and the MSC. The handshaking is done through wireline link which is assumed to be safely guarded. In other words we are assuming that wireline communication links are secure. We shall concentrate only on the security of radio links.

In this paper, we propose two authentication protocols: one for call setup and one for handoff. Our protocols allow a MU and a BS to verify each other. It also offers an additional feature of providing MUs with location privacy. Featuring two-way authentication and location privacy are particularly desired in a mobile communication network. There are in general two kinds of authentication scheme: one-way and two-way. One-way authentication is only suitable when sender completely trusts the receiver, whereas in mobile environment, MUs and BSs do not know each other (i.e. the two parties do not each other completely trust). Location privacy is a protection against tracing the location of a mobile unit by listening to the signalling exchanged on the radio link. The protection is normally done by the use of random number. In our scheme a random number is generated each time a new call is established. A random number was given and stored into a mobile unit when it registered with MSC.

For the convenience of describing our work, we first define the following set of symbols:

1. $M U_{i}$ : The $i$ th mobile unit.

2. $B S_{i}$ : The $i$ th base station.

3. $R C$ : A random check number, different $R C$ 's are used in different call setups. $R C$ is chosen randomly by MSC at each call setup and given to $M U_{i}$.

4. $K_{c}$ : The conference key used by $M U_{i}$. The regular messages including voice and data are encrypted by $K_{c}$ after a conference call is correctly built.

5. $K_{s}$ : The session key generated by $M U_{i}$. It is chosen by $M U_{i}$ at each call setup and used to participate in the construction of $K_{c}$.

6. $P K C$ : Public key cryptosytem. In our protocol we use low exponent RSA as our basic cryptosystem of which public keys are $n=p q$ and $e$, the secret key is $d$, where $p, q$ are two large primes. Encryption key $e$ and decryption key $d$ must satisfy $e d \equiv 1(\bmod (p-$ 1) $(q-1))$. MSC first chooses a primitive root $g$ which falls in both $G F(p)$ and $G F(q)$ and makes it public. In order to satisfy the low-circuit complexity and fast computation requirement desired for a mobile unit, the encryption exponent $e$ is chosen to be a small integer. Here, we let $e=3$ and both $p$ and $q$ be about 256 bits long. $d$ is obtained by satisfying the relation $3 \times d \equiv 1(\bmod (p-1)(q-1))$.

7. $h(\bullet)$ : one-way hash function.

8. $a \| b$ : a concatenation operation for $a$ and $b$. 


\subsection{Call Setup Authentication Protocol}

This protocol is executed each time a call setup request is launched. The protocol is sketched in Fig. 1 and described as follows.

Step 1. $M U_{i}$ encrypts $\left(I D_{M U_{i}}\|R C\| K_{s}\right)$ by using $P K C$ of MSC and computes $C_{M U_{i}}=$ $\left(I D_{M U_{i}}\|R C\| K_{s}\right)^{3} \bmod n$, then sends $C_{M U_{i}}$ to $B S_{i}$ via the raido link.

Step 2. After receiving $C_{M U_{i}}, B S_{i}$ forwards the received $C_{M U_{i}}$, and his identity $I D_{B S_{i}}$ to MSC.

Step 3. MSC receives $I D_{B S_{i}}$, descrypts $C_{M U_{i}}$ and gets $\left(I D_{M U_{i}}\|R C\| K_{s}\right)$. MSC searches the identity of $M U_{i}$, if $I D_{M U_{i}}$ does exist in the database, MSC further compares the $R C$ sent from $M U_{i}$ to see if it is identical to $R C$ prestored in the database. If the comparison is correct, $M U_{i}$ is accepted as a legal subscriber and go to the next step; otherwise, MSC terminates the call setup procedure.

Step 4. MSC selects randomly a new random check number $R C^{\prime}$ for $M U_{i}$ to use during the next call setup. MSC then computes a number $N R=R C \oplus R C^{\prime}$, where " $\oplus$ " is an invertible operator.

Step 5. MSC generates a secret key $S_{B S_{i}}^{(0)}$ for $B S_{i}$ as $S_{B S_{i}}^{(0)}=\left(h\left(I D_{B S_{i}}\right) \times R C\right)^{d} \bmod n$ sends $\left\{S_{B S_{i}}^{(0)}, N R\right\}$ to the $B S_{i}$ of $M U_{i}$.

Step 6. $B S_{i}$ chooses a random number $r_{i}$, generates

$$
\begin{gathered}
X_{B S_{i}}=g^{-3 r_{i}} \bmod n, \\
Y_{B S_{i}}=S_{B S_{i}^{(0)}} \times g^{r_{i}} \bmod n,
\end{gathered}
$$

and sends $\left\{I D_{B S_{i}}, X_{B S_{i}}, Y_{B S_{i}}, N R\right\}$ to $M U_{i}$

Step 7. After receiving $\left\{I D_{B S_{i}}, X_{B S_{i}}, Y_{B S_{i}}, N R\right\}$ from $B S_{i}, M U_{i}$ computes

$$
\frac{Y_{B S_{i}}^{3} \times X_{B S_{i}}}{R C} \bmod n
$$

to see if it is equal to $h\left(I D_{B S_{i}}\right)$, where $I D_{B S_{i}}$ is sent from $B S_{i}$ and $R C$ is a random number prestored in $M U_{i}$. If they do match then $B S_{i}$ is treated legal and go to the next step; otherwise $M U_{i}$ hangs up the call.

Step 8. $M S_{i}$ sends an acknowledgement to $B S_{i}$ and $B S_{i}$ tells MSC the acknowledgement via the wireline network.

Step 9. $M U_{i}$ replaces $R C$ with $R C^{\prime}$ for the next call setup and assigns a number $V_{B S_{i}}^{(0)}=$ $h\left(I D_{B S_{i}}\right)$ for use in handoff.

Step 10. After receiving the acknowledgement from $M U_{i}$, MSC replaces $R C$ in the database with $R C^{\prime}$ and stores $S_{B S_{i}}^{(0)}$ for use in handoff, the two-way authentication procedure is now completed.

\subsection{Secure Conference Call Construction}

In this section we present a secure conference call for mobile communications. We assume that there are $m+1$ mobile units $M S_{0}, M S_{1}, M S_{2}, \ldots, M S_{m}$ in the system. Let $M S_{0}$ be the chairperson who initiates the electronic conference with any $k$ of the other $m$ mobiles, where $1 \leq k \leq m$. Let $G=\left\{M U_{i_{1}}, M U_{i_{1}}, \ldots, M U_{i_{k}}\right\}$ be the set of conferees to whom $M U_{0}$ wishes to establish connection. The conference call prototol is sketched in Fig. 2 and summarized as follows. 
Step 1. $M U_{0}$ computes $\left(I D_{M U_{0}}\left\|R C_{0}\right\| K_{s_{0}}\right)^{3} \bmod n$ and

$\left(I D_{M S_{i_{1}}}\left\|I D_{M S_{i_{2}}}\right\| \ldots \| I D_{M S_{i_{k}}}\right)^{3} \bmod n$ and sends them to MSC via $B S_{0}$.

Step 2. $M S_{0}$ and MSC authenticate each other using our authentication protocol for call setup. If the authentication is successfully completed, then MSC calls the other $M U_{i_{j}}, j=$ $1,2, \ldots, k$ in $G$.

Step 3. After receiving the call signal, each $M U_{i_{j}}$ computes and sends $\left(I D_{M U_{i j}}\left\|R C_{i_{j}}\right\| K_{s_{i_{j}}}\right)^{3}$ to MSC via $B S_{i_{j}}$.

Step 4. Both $M S_{i_{j}}$ and $B S_{i_{j}}$ authenticate each other using our call setup authentication protocol. After the authentication between $M S_{i_{j}}$ and $B S_{i_{j}}$ is completed, then $M U_{i_{j}}$ now shares a common session key $K_{s_{i}}$ with MSC.

Step 5. MSC collects the coordinate points $\left(I D_{M U_{0}}, K_{\mathbf{s}_{0}}\right)$ and $\left(I D_{M U_{i_{j}}}, K_{s_{i_{j}}}\right), j=1,2, \ldots, k$ and then constructs an interpolating polynomial $f(z)$ of degree $k$ over $G F(n)$ passing through these coordinate points.

Step 6. MSC generates a number $K_{c}=f(0)$ as the common encryption key in the conference, regular messages including voice and data are encrypted by $K_{c}$ during the conference.

Step 7. MSC selects $k$ distinct coordinate points $\left(a_{j}, b_{j}\right), j=1,2, \ldots, k$ from the polynomial $f(z)$.

Step 8. MSC broadcasts $\left(a_{j}, b_{j}\right), j=1,2, \ldots, k$ to the participating mobile units of the conference.

Step 9. Each participating mobile unit in the conference individually reconstructs the interpolating polynomial $f(z)$ of degree $k$ over $G F(n)$ via the $k$ known coordinate points $\left(a_{j}, b_{j}\right), j=1,2, \ldots, k$ and his own coordinate pair $\left(I D_{M U}, K_{s}\right) . K_{c}$ is thus obtained by substituting $z=0$ into $f(z)$.

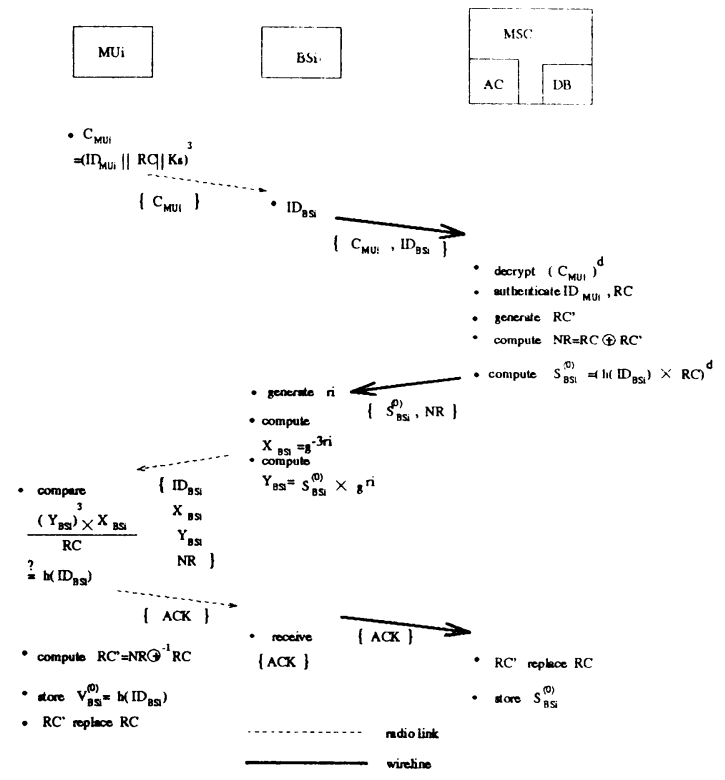

Fig. 1 Call setup authentication protocol 


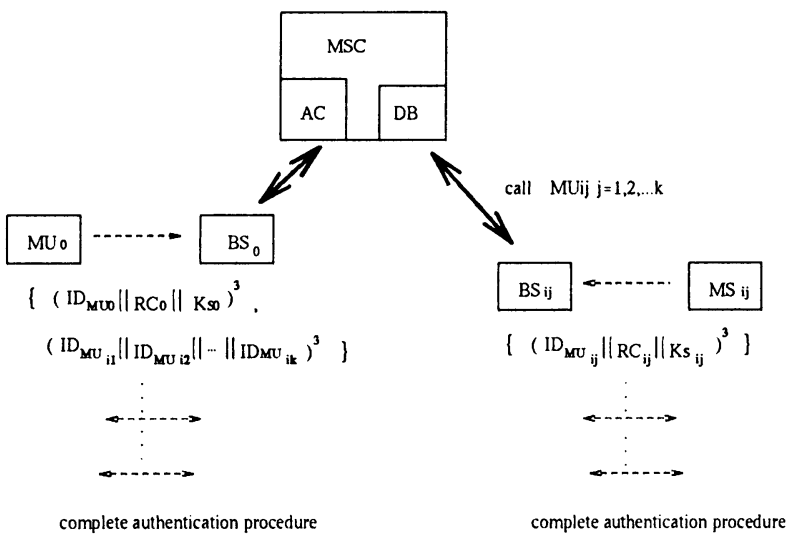

(a) Two-way authentication

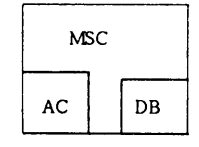

- collect ( ID $\mathrm{MuO}_{\mathrm{O}} . \mathrm{Kg}_{\mathrm{g}}$ ). $\left(\mathrm{ID}_{\mathbf{M U}}{ }_{\mathrm{ij}}, \mathrm{Ks}{ }_{\mathrm{ij}}\right) \mathrm{j}=1,2, \ldots \mathrm{k}$ - construct $f(z)$. let $K c=f(0)$

- generate other (aj,bj) $j=1,2, \ldots . k$ on loci of $f(z)$

- broadcast (aj,bj) $j=1,2, \ldots . k$

(b) Construction of $\mathrm{Kc}$

Fig. 2 Conference call construction

\subsection{Handoff Authentication Protocol}

When $M U_{i}$ moves across the cell boundary, it needs to switch from the old base station, $B S_{i}$, to the new base station, $B S_{i-n e w}$. This procedure is known as handoff. During handoff, it is possible that an intruder pretends to be a genuine base station. In the following we propose an authentication protocol against such attack during handoff. Suppose that initially $M U_{i}$ is communicating with $B S_{i}$ via the radio channel. When the first handoff occurs $B S_{i-n e w}$ sends a new secret key $S_{B S_{i}}^{(1)}$ to $M U_{i}, M U_{i}$ reauthenticates the new base station $B S_{i-n e w}$. The protocol is sketched in Fig. 3 and described as follows.

Step 1. MSC chooses a new basebase $B S_{i-\text { new }}$, computes $S_{B S_{i}}^{(1)}=\left(h\left(I D_{B S_{i-\text { new }}}\right)\right)^{d} \times S_{B S_{i}}^{(0)}$, where $I D_{B S_{i-\text { new }}}$ is the identity of $B S_{i-n e w}, S_{B S_{i}}^{(1)}$ is next sent to $B S_{i-n e w}$.

Step 2. After receiving $S_{B S_{i}}^{(1)}, B S_{i-n e w}$ chooses a random number $r_{i}^{\prime}$, generates

$$
\begin{gathered}
X_{B S_{i-\text { new }}}=g^{-3 r_{i}^{\prime}} \bmod n, \\
Y_{B S_{i-\text { new }}}=S_{B S_{i}}^{(1)} \times g^{r_{i}^{\prime}} \bmod n .
\end{gathered}
$$

and sends $\left\{I D_{B S_{i-\text { new }}}, X_{B S_{i-\text { new }}}, Y_{B S_{i-\text { new }}}\right\}$ to $M U_{i}$

Step 3. After receiving $\left\{I D_{B S_{i-\text { new }}}, X_{B S_{i-\text { new }}}, Y_{B S_{i-\text { new }}}\right\}$ from $B S_{i-n e w}, M U_{i}$ computes

$\frac{Y_{B S_{i-\text { new }}^{3}}^{3} \times X_{B S_{i-\text { new }}}}{R C} \bmod n$ to see if it is equal to $V_{B S_{i}}^{(0)} \times h\left(I D_{B S_{i-\text { new }}}\right)$, where $V_{B S_{i}}^{(0)}$ is prestored in $M U_{i}, I D_{B S_{i-n e w}}$ is sent from $B S_{i-n e w}$ and $R C$ is a random number prestored in $M U_{i}$. If they do match then $B S_{i-n e w}$ is accepted by $M U_{i}$ as a legal base station.

Step 4. After completing the handoff procedure, $M U_{i}$ stores $V_{B S_{i}}^{(1)}=V_{B S_{i}}^{(0)} \times h\left(I D_{B S_{i-\text { new }}}\right)$ and MSC stores $S_{B S_{i}}^{(1)}$. 
Due to the new base station and the previous base station are connected with MSC through wireline links, and the wireline links are made secure in our assumption so that a legal signature of new base station must be relative to previous one. Therefore, if the handoff procedure is executed $l$ times for a $M U_{i}$ after the conference call is built, the secret key $S_{B S_{i}}^{(l)}$ of new base station $B S_{i-\text { new }}$ is then computed as $S_{B S_{i}}^{(l)}=\left(h\left(I D_{B S_{i-n e w}}\right)\right)^{d} \times S_{B S_{i}}^{(l-1)}$ and $V_{B S_{i}}^{(l)}=V_{B S_{i}}^{(l-1)} \times$ $h\left(I D_{B S_{i-\text { new }}}\right), l \geq 1$.

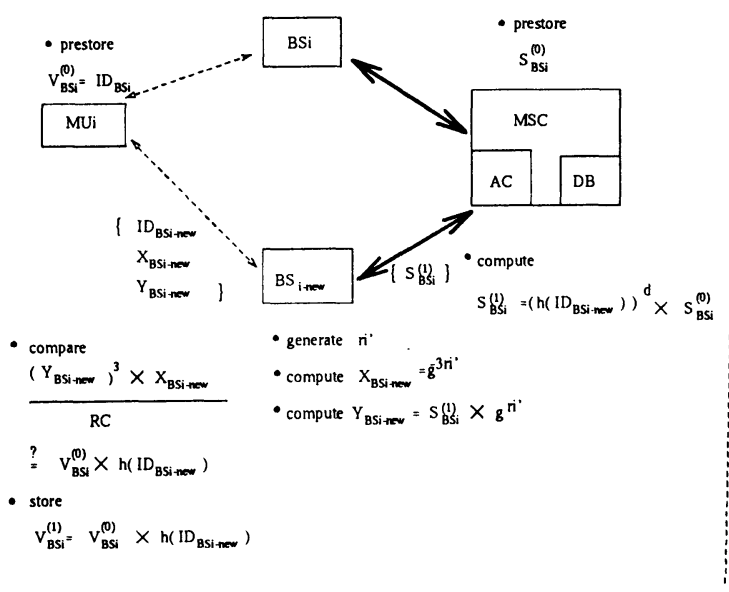

When the handoff procedure is executed limes for a MUi, $l \geqq$

- prestore

$\mathrm{v}_{\mathrm{BSi}}^{(-1)}$

MUi

- compare

$\frac{\left(Y_{B S i \text { new }}\right)^{3} \vee X_{\text {RSi now }}}{R C}$

$\stackrel{?}{=} \mathrm{V}_{\mathrm{BSi}}^{(\cdot \cdot l)} \times \mathrm{h}\left(\mathrm{ID}_{\mathrm{BSi}+\mathrm{now}}\right)$

tore

$\mathrm{V}_{\mathrm{BSi}}^{(j)}=\mathrm{V}_{\mathrm{BSi}}^{(-1)} \times \mathrm{x}\left(\mathrm{ID}_{\mathrm{BS}-\text { now }}\right)$

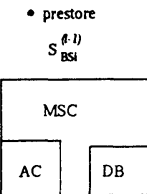

compute

$\mathrm{S}_{\mathrm{BSi}}^{(i)}=\left(\mathrm{h}\left(\mathrm{ID}_{\mathrm{BSi}-\mathrm{new}}\right)\right)^{d} \times \mathrm{S}_{\mathrm{BSI}}^{(-1)}$

Fig. 3 Handoff authentication protocol

\section{DISCUSSIONS AND SECURITY ANALYSIS}

Our scheme offers the following attractive features.

1. Message confidentiality: The sensitive information is well protected from outsiders during the handshaking between a subscriber and a BS via the radio link. After the conference call is correctly built, the regular messages including voice and data can also be well protected from outsiders by using the common conference key $K_{c}$. $K_{c}$ is reconstructable by all the authorized mobiles' session keys $K_{s}$ 's, and $K_{s}$ 's are secure under the use of RSA scheme, Rivest et al. (1978).

2. Location privacy: Users such as wealthy people or high ranking government officials may desire to keep their location secret since location is a critical portion of safety. Location privacy is achieved through the use of random check in our protocol.

3. Two-way confirmation: The first generation cellular mobile systems, such as AMPS, provides only voice service so that only the invasion from an illegal subscriber is concerned. 
But in the second or third generation of cellular mobille systems which will provide data and other types of service, the impersonation of a subscriber or a genuine BS is possible. Thus two-way authentication becomes necessary and important. Our protocol obviously can provide two-way authentication during call setup. The security of our authentication scheme relies on the difficulty of forging signatures $X_{B S}$ and $Y_{B S}$ which satisfy (1) and (2), since the system secret key $d$ must be first evaluated, it seems intractable as in the well-known RSA.

4. Authentication during handoff: In handoff, the communication link from a subscriber to the old BS is first terminated and switched to a new link to the new BS. During this transition period, it is possible for an illegal BS to personate a new genuine base station. Our protocol indeed prevents this from happening. Since the signature message of new BS is computed from the secret key $S_{B S}$ of old BS and $S_{B S}$ is transmitted by MSC via secure wireline.

5. Security of the conference key: During the conference, in order to achieve secrecy, the message has to be encrypted under $K_{c}$ before sending out through the radio channel. For $K_{c}$, it can only be construced by the authorized mobiles who participate in the conference. Consider a polynomial $f(z)$ of degree $k$ which is constructed by interpolating $k+1$ distinct coordinate points $\left(I D_{M U_{0}}, K_{s_{0}}\right)$ and $\left(I D_{M U_{i j}}, K_{s_{i j}}\right), j=1,2, \ldots, k$. MSC chooses other $k$ distinct coordinate points $\left(a_{j}, b_{j}\right), j=1,2, \ldots, k$ from the loci of $f(z)$. Each $M U$ interpolates the $k$ coordinate points $\left(a_{j}, b_{j}\right) \mathrm{s}$ with his own coordinate point $\left(I D_{M U}, K_{s}\right)$ to obtain a polynomial $\hat{f}(z)$, then $\hat{f}(z)$ is identical to $f(z)$. Owing to $K_{\mathrm{s}}$ is only known by an authorized $M U$ in the conference, $K_{c}$ can be obtained from $f(z)$ with $z=0$. But other unauthorized $M U$ s have no way of getting $K_{c}$. Therefore, $K_{c}$ is secure from revealing by outsiders.

6. Free of replay: Replay is a serious problem in authentication protocol. An easy solution is to embed a time stamp into the message, while time synchronization is needed for the time stamp strategy. In our scheme, because random number $R C$, signature pair $\left(X_{B S}, Y_{B S}\right)$ are randomly changed in each authentication procedure, the attack of replay can thus be avoided.

7. Low computation complexity: Due to the requirements of low circuit complexity and low power consumption in handset, a subscriber has only limited computing power. The computation complexity of exponent encryption in our scheme would be lower when a small exponent (e.g. $e=3$ ) is to be chosen and $n$ is a 512-bit composite number. Using the addition chain method in Knuth (1981), only two modulo multiplications are required. In an 8-bit microcontroller, handset or low-circuit complexity terminals, for example, the computation time in a single 512 modulo multiplication takes about $0.1 \mathrm{~s}$ so that our handset only requires about $0.2 \mathrm{~s}$ processing time in performing the pubic key encryption. Using RSA scheme, approximately $20 \mathrm{~s}$ is needed for an 8-bit microcontroller. On the other hand, the construction of $K_{c}$ in each $M U$ needs $O\left(k \log ^{2} k\right)$ operations using methods discussed in Knuth (1981), where $k$ is the total number of participating mobile units in the conference call. It is believed that a mobile can perform such computation in a reasonable time.

8. Defend the intrusion from BS: In a cellular mobile system, there are many widespread BSs. These BSs are unprotected and may be intruded. Anyone trying to steal the secret information from subscribers can be done by intruding a BS. So it is insecure to place permanent and secret information in BS. A MSC is usually more well protected to have better protection of permanent and secret information. In our protocol there is no permanent and secret information stored in BS. What placed in the BS, are temporary messages. Even when an intruder can collect all the information of a specific user in BS, he still can 
not determine the secret $d$ of MSC since breaking the system is the same as challenging the RAS algorithm.

One concern of our work is that the MSC needs to maintain a database of all the home subscribers' relative information which may be vulnerable to attacks. But this can be prevented by storing these information in ciphertext form.

\section{CONCLUSIONS}

In this paper we have proposed an ID-based two-way authentication scheme suitable for digital cellular mobile radio system in terms of hardware complexity, time complexity and power consumption. The proposed scheme is initially finished through two-way authentication, and further extented to multi-mobile unit in order to hold a conference call. In particular, the secret information appearing in BSs is randomly changed to prevent the intruder from penetrating BSs to steal the information. Although the computation in MSC is more complex, our assumption that MSC has powerful computing capability to compute the secret key of BS in the manner that a subscriber and a BS can authenticate each other within a reasonable time is not too unrealistic.

\section{REFERENCES}

Ashar A. and Diffie, W. (1994) Privacy and Authentication for Wireless Local Area Networks. IEEE Personal Communications, First Quarter, 25-31.

Beller, M.J., Chang L.F. and Yacobi, Y. (1993) Privacy and Authentication on a Portable Communications System. IEEE J. Select. Areas. Commun, vol. SAC-11, 821-829.

Coomaraswamy G. and Kumar, S.P.R. (1993) A Novel Method for Key Exchange and Authentication with Cellular Network Applications. IEEE Conference, ICUPC'93, 186-190.

Diffie, W. and Hellman, M.E. (1976) New Directions in Cryptography. IEEE Trans. Information Theory, vol. 22, no. 6, 644-654.

Haug, T. (1994) Overview of GSM: Philosophy and Results. Int. Journal of Wireless Information Networks, vol. 1, 7-16.

Hwang, M.S. and Yang, W.P. (1995) Conference Key Distribution Protocols for Digital Mobile Communication Systems. IEEE J. Select. Areas Commun., vol. 13, no. 2, 416-420.

Hwang, T. (1993) Scheme for Secure Digital Communication Based on Symmetric Key Cryptography. Information Processing Letters, vol. 48, 35-37.

Knuth, D.E. (1981) The Art of Computer Programming-Seminumerical Algorithms, vol. 2. Addision-Wesley, Reading, MA.

Lin, H.Y. and Harn, L. (1993) Authentication in Wireless Communications. IEEE Conference, Globlem Com'93, 550-554.

Molva, R, Samfat, D. and Tsudik, G. (1994) Authentication of Mobile Users. IEEE Network, March/April, 26-34.

Okamota, E. and Tanaka, K. (1989) Key Distribution System Based on Identification Information. IEEE J. Select. Areas Commun., vol. 7, no. 4, 481-485.

Rivest, R.L., Shamir, A. and Adleman, L. (1978) A Method for Obtaining Digital signatures and Public-key Cryptosystems. Comm. of ACM, vol. 21, no. 2, 120-126.

Shamir, A. (1985) Identity-Based Cryptosystems and Signature Schemes. Advances in Cryptology: Proceedings of Crypto'84, Springer-Verlag, 47-53. 
T1P1.3/93-044R1. (1993) Draft technical report on privacy and authentication objectives for wireless access to personal communications, May, 9-11.

Tanenbaum, A.S. (1988) Computer Networks. Englewood, New Jersey: Prentice Hall, Second ed.

Tatebayashi, M., Matsuzaki N. and Newman, D.B. (1990) Key Distribution Protocol for Digital Mobile Communication Systems. Advances in Cryptology: Proceedings of Crypto'89, Springer-Verlag, 324-333.

Tsujii, S. and Itoh, T. (1989) An ID-Based Cryptosystem Based on the Discrete Logarithm Problem. IEEE J. Select. Areas Commun., vol. 7, no. 4, 467-473.

\section{BIOGRAPHY}

Shiuh-Jeng Wang was born in Taiwan, 1967. He received the M.S. degree in Applied Mathematics from National Chung-Hsing University, Taichung, Taiwan, Republic of China in 1991.

He is currently working toward the Ph.D. degree in Electrical Engineering at the National Taiwan University, Taipei, Taiwan. His research interests include cryptography, authentication in mobile communications and data structure.

Le-Pond Chin was born in Tainan, 1962. He received the B.S. degree in telecommunication engineering from the National Chiao-Tung University, Hsing-Chu, Taiwan, in 1984, and the M.S. and Ph. D. degrees in electrical engineering from the National Taiwan University, Taipei, Taiwan in 1989 and 1995, respectively.

$\mathrm{He}$ is with the faculty of the Shih-Chien College, Taipei,Taiwan from 1989, and currently directs the Computer Center of the Shih-Chien College. His research fields include mo$\mathrm{bile/satellite} \mathrm{communications,} \mathrm{integrated} \mathrm{voice/data} \mathrm{transmission,} \mathrm{and} \mathrm{security.}$

Jin-Fu Chang was born in Taiwan, 1948. He received the B.S.E.E. degree from the National Taiwan University, Taipei, in 1970, and the Ph.D. degree in Electrical Engineering and Computer Sciences from the University of California, Berkeley, in 1977.

He has been a Professor of Electrical Engineering at National Taiwan University, Taipei, Taiwan, since August 1982. He was also the Department Chairman from August 1985 to July 1987 and then on leave at the Ministry of Education as Director of Science and Technology Advisory Office for three years from July 1987 to June 1990. He spent three years (August 1991 - July 1994) at the National Central University as Provost. His research interests include computer communications, high-speed networks, performance analysis, wireless communications and cryptography. He is a Fellow, IEEE.

Ywh-Ren Tsai was born in Kaohsiung, Taiwan, 1967. He received the B.S. degree in electrical engineering from National Tsing-Hua University, Hsing-Chu, Taiwan, in 1989, and the $\mathrm{Ph} . \mathrm{D}$. degree in electrical engineering from the National Taiwan University, Taipei, Taiwan in 1994.

He is currently with the Laboratory of Transmission Technology, Telecommunication Laboratories, Directorate General of Telecommunications, Taiwan, as an Associate Researcher. His research interests include spread spectrum communications, mobile communications, cryptography and coding theory. 